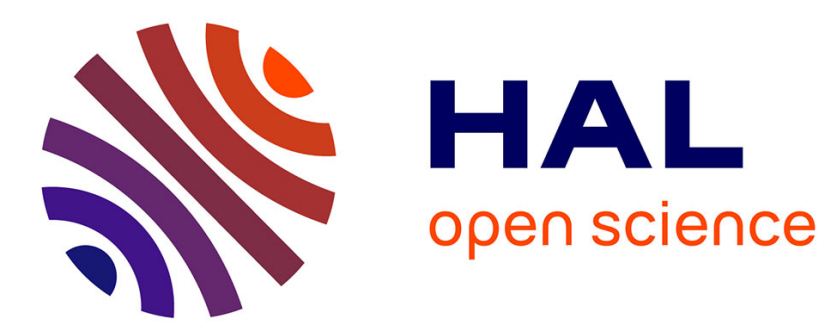

\title{
Remarques sur les aberrations des lentilles électrostatiques unipotentielles accélératrices
}

\author{
G. Slodzian, A. Figueras
}

\section{To cite this version:}

G. Slodzian, A. Figueras. Remarques sur les aberrations des lentilles électrostatiques unipotentielles accélératrices. Journal de Physique Lettres, 1978, 39 (7), pp.90-93. 10.1051/jphyslet:0197800390709000 . jpa-00231450

\section{HAL Id: jpa-00231450 https://hal.science/jpa-00231450}

Submitted on 1 Jan 1978

HAL is a multi-disciplinary open access archive for the deposit and dissemination of scientific research documents, whether they are published or not. The documents may come from teaching and research institutions in France or abroad, or from public or private research centers.
L'archive ouverte pluridisciplinaire HAL, est destinée au dépôt et à la diffusion de documents scientifiques de niveau recherche, publiés ou non, émanant des établissements d'enseignement et de recherche français ou étrangers, des laboratoires publics ou privés. 


\title{
Classification \\ Physics Abstracts \\ 07.80 \\ REMARQUES SUR LES ABERRATIONS DES LENTILLES ÉLECTROSTATIQUES UNIPOTENTIELLES ACCÉLÉRATRICES
}

\author{
G. SLODZIAN et A. FIGUERAS (*)
}

Laboratoire de Physique des Solides (**)

Université de Paris-Sud, Bât. 510, 91405 Orsay Cedex, France

(Reçu le 12 juillet 1977, révisé le 30 janvier 1978, accepté le 21 février 1978)

\begin{abstract}
Résumé. - On compare les aberrations de sphéricité et de chromaticité d'une lentille électrostatique symétrique à trois électrodes lorsqu'elle fonctionne suivant un mode soit accélérateur, soit retardateur. Le premier type de fonctionnement conduit à des aberrations plus faibles. Les montages optiques utilisant des particules issues d'émissions secondaires, la production de sondes fines, pourraient bénéficier des propriétés optiques des lentilles accélératrices.
\end{abstract}

\begin{abstract}
Chromatic and spherical aberrations from a three aperture symmetrical electrostatic lens are compared in two working modes, an accelerating and a retarding mode. It is concluded that the first mode leads to lower aberrations. Optical systems acting on secondary emitted particles or producing small probes could benefit from the optical properties of accelerating lenses.
\end{abstract}

1. Introduction. - Dans les montages d'optique ionique les seules lentilles que l'on utilise en pratique sont des systèmes électrostatiques. Parmi ces systèmes, les lentilles unipotentielles à trois électrodes sont d'un emploi très courant. La figure 1 donne le schéma d'un ensemble symétrique formé par trois diaphragmes plans, percés d'un trou circulaire de diamètre $D$ centré sur l'axe $z^{\prime} z$. Les deux électrodes externes sont au même potentiel $V_{2}$, l'électrode centrale est portée au potentiel $V_{1}$. Par convention, on prend $V_{2}$ égal à

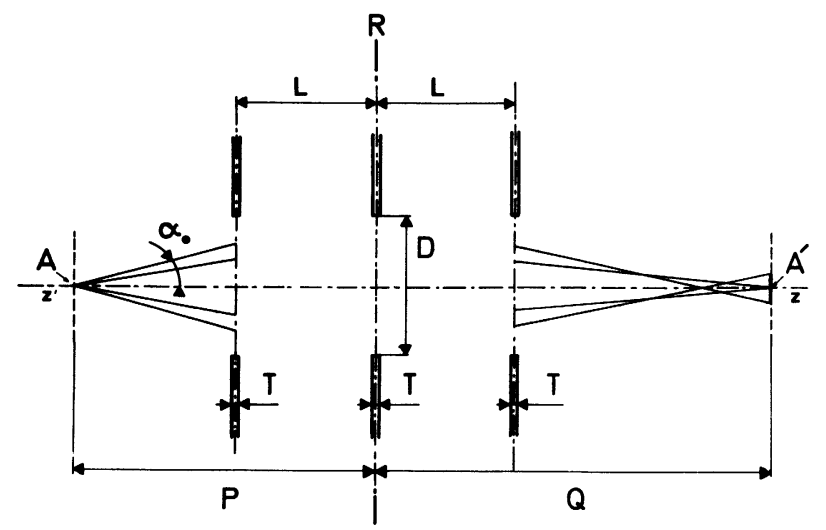

Fig. 1. - Le cas étudié correspond à $L / D=1$ et $T / D=0,05$.

[The case studied corresponds to $L / D=1$ and $T / D=0.05$.]

(*) Instituto de Quimica Organica, Barcelona, Espagne. (**) Associé au C.N.R.S. l'énergie cinétique des ions, exprimée en électronvolts, avant leur entrée dans la zone d'action de la lentille.

Entre l'électrode d'entrée et le plan médian $\mathbf{R}$ de l'électrode centrale, les particules sont soit freinées, soit accélérées suivant le signe du potentiel $V_{1}$ et celui des particules, dans les deux cas la lentille est convergente; dans le premier cas, on dit qu'elle est décélératrice, dans le second qu'elle est accélératrice. Dans cette note, nous nous proposons d'attirer l'attention sur une propriété peu connue des lentilles unipotentielles accélératrices à savoir que leurs aberrations de sphéricité et de chromaticité sont plus faibles que celles des lentilles de mểme géométrie fonctionnant suivant un mode retardateur avec la même longueur focale. Pour des raisons de simplicité, nous nous limiterons à une seule géométrie, celle qui est décrite sur la figure 1 .

2. Aberration de sphéricité. - Prenons le plan médian $\mathrm{R}$ comme plan de référence. La lentille donne de l'objet ponctuel A situé sur l'axe à une distance $P$ de $\mathrm{R}$, une image gaussienne $\mathrm{A}^{\prime}$ située à une distance $Q$. Les trajectoires issues de $A$ remplissent un cône de sommet $\mathrm{A}$ et de demi-angle d'ouverture égal à $\alpha_{0}$. En fait au lieu d'aller converger en $A^{\prime}$, elles viennent percer le plan de front passant par $\mathrm{A}^{\prime}$ et remplir un disque d'aberration dont le rayon $\Delta r=M C_{\mathrm{S}} \alpha_{0}^{3}$; $M$ est le grandissement linéaire de l'image en $\mathrm{A}^{\prime}$ et $C_{\mathrm{s}}$ le coefficient d'aberration de sphéricité. 
En utilisant la relation $\alpha_{0}=M \alpha_{i} ; \alpha_{i}$ angle que fait avec l'axe la trajectoire " $\alpha_{0}$ " après la traversée de la lentille, on peut encore écrire : $\Delta r=M^{4} C_{\mathrm{S}} \alpha_{i}^{3}$.

Il a été montré [1] que le coefficient $C_{\mathrm{s}}$ pouvait se mettre sous la forme

$$
C_{\mathrm{S}}=\sum_{n=0}^{4} C_{\mathrm{S} n} M^{-n}
$$

et l'on trouve dans la littérature des valeurs des coefficients $C_{\mathrm{S} n}$ correspondant à différentes géométries.

Pour comparer les deux types de fonctionnement, nous nous limiterons au cas où l'objet est à l'infini. Le point $\mathrm{A}^{\prime}$ tend alors vers le foyer image $\mathrm{F}$ et $Q$ tend vers l'abscisse $Z_{\mathrm{F}}$ du plan focal. Il en résulte que $\Delta r \simeq C_{\mathrm{S} 4} \alpha_{i}^{3}$.

Sur la figure 2, nous avons tracé le graphe $C_{\mathrm{S} 4}$ en fonction de $f / D$ pour les deux modes de fonctionnement de la lentille. De plus, les graphes de $f / D$ fonction de $v=V_{1} / V_{2}$ pour les deux modes (Fig. $3 a, b$ ) permettent de préciser le rapport des tensions nécessaire pour avoir une distance focale déterminée. Les tracés ont été effectués en utilisant les valeurs données par Harting et Read [1].

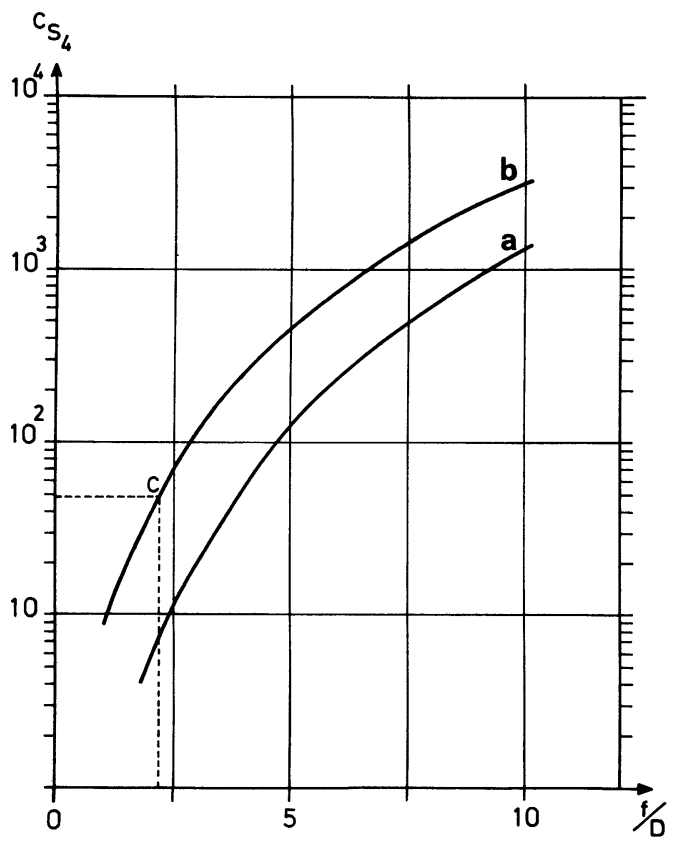

FIG. 2. - $\mathrm{a}$ : Système accélérateur; $\mathrm{b}$ : Système décélérateur ; $c$ : Limite de surexcitation $(v=0)$.

[a : Accelerating mode. b : Retarding mode. $\mathrm{c}$ : Overexcitation point $(v=0)$.]

L'examen de ces graphes montre que l'aberration de sphéricité est toujours plus faible pour la lentille accélératrice. Ainsi, pour une longueur focale $f / D$ de l'ordre de 3, le coefficient d'aberration de sphéricité est 6 fois plus petit. Des résultats analogues ont été obtenus pour d'autres valeurs de $\boldsymbol{M}$.
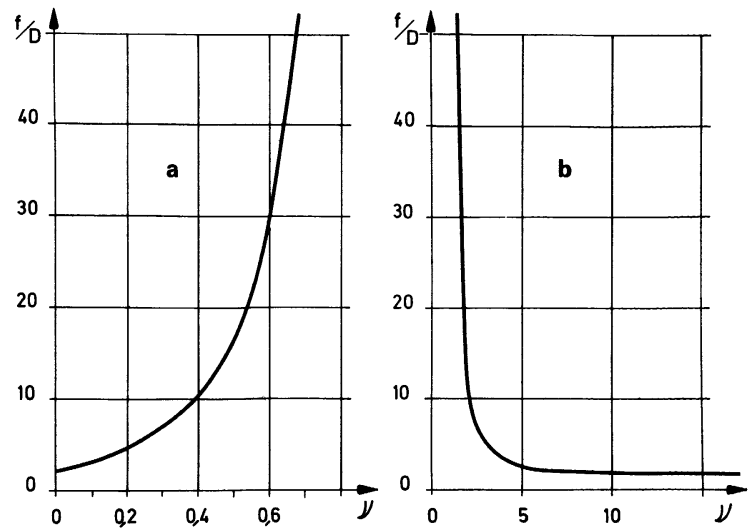

FIG. 3. - a) Lentille retardatrice ; $b$ ) Lentille accélératrice.

[a) Retarding mode. $b$ ) Accelerating mode.]

Il convient toutefois de remarquer que la géométrie très ouverte que nous examinons ici conduit à des graphes $f / D$ fonction de $v$ qui ne sont pas les plus typiques. D'autres géométries, plus fermées, font apparaître un minimum dans les graphes $f / D$ fonction de $v$ auquel correspond des propriétés optiques intéressantes [3].

D'un point de vue qualitatif, on peut dire que la diminution de l'aberration sphérique est liée, en partie, à ce que, lors de la traversée d'une lentille accélératrice, les trajectoires restent comparativement plus près de l'axe. L'examen de l'expression du coefficient d'aberration sphérique donnée par l'intégrale de Scherzer [4] tend à confirmer ce point de vue. En effet, l'intégrale porte sur des fonctions où $r(z)$, solution de l'équation gaussienne des trajectoires donnant la distance à l'axe d'une trajectoire méridienne particulière, figure à des puissances élevées - mais il faut aussi remarquer que l'énergie des particules joue également un rôle dans la diminution de l'aberration puisque cette énergie apparaît à des puissances élevées aux dénominateurs des fonctions à intégrer -

Ces considérations sont à rapprocher des résultats obtenus avec les lentilles retardatrices asymétriques ou de formes hyperboliques [2] pour lesquelles la réduction de l'aberration sphérique est due, en partie, à ce que la trajectoire $r(z)$ est plus proche de l'axe dans les régions où la convergence de la lentille est forte.

3. Aberration chromatique. - Négligeons l'aberration de sphéricité et examinons le devenir d'une trajectoire incidente parallèle à l'axe lorsque celle-ci est parcourue par des particules dont les énergies, exprimées en électron-volts, diffèrent d'une petite quantité $\Delta U$.

Nous savons que la position du foyer image $Z_{\mathrm{F}}$ et la longueur focale $f$ dépendent du rapport $v$. Comme $V_{2}$ mesure l'énergie d'une particule incidente, tout se passe comme si la particule d'énergie $V_{2}+\Delta U$ entrait avec une énergie $V_{2}$ dans une 
lentille dont les électrodes externes et l'électrode centrale seraient respectivement aux potentiels

$$
V_{2}-\Delta U \text { et } V_{1}-\Delta U
$$

Cette particule voit donc la lentille comme si le rapport $v$ avait été changé de

$$
\Delta v=(v-1) \frac{\Delta U}{V_{2}} .
$$

A ce changement de $v$ correspond une variation :

$$
\Delta Z_{\mathrm{F}} \frac{\mathrm{d} Z_{\mathrm{F}}}{\mathrm{d} v}(v-1) \frac{\Delta U}{V_{2}}
$$

Or le faisceau de trajectoires parallèles contenues dans le cylindre de rayon $h$ est parcouru par des particules dont les énergies sont dispersées dans une bande d'intervalle $\left(V_{2}-\Delta U, V_{2}+\Delta U\right)$. Ce faisceau forme, autour de $\mathrm{F}$, une tache d'aberration de rayon $\Delta r=\Delta Z_{\mathrm{F}} \alpha_{i}$ avec $\alpha_{i}=h / f$.

L'expression de $\Delta r$ s'écrit encore :

$$
\Delta r=h G_{\mathrm{c}} \frac{\Delta U}{V_{2}}
$$

où le coefficient sans dimension

$$
G_{\mathrm{c}}=(f / D)^{-1} \frac{\mathrm{d}}{\mathrm{d} v}\left(Z_{\mathrm{F}} / D\right)(v-1)
$$

permet de caractériser l'aberration chromatique. Le calcul de $G_{\mathrm{c}}$ en fonction de $v$ peut se faire soit à partir d'expressions analytiques de $Z_{\mathrm{F}} / D$ et $f / D$ comme celles données par Regenstreiff [3], soit en utilisant les valeurs des éléments cardinaux publiés dans la littérature [5].

Sur la figure 4 , nous avons tracé les graphes de $G_{\mathrm{c}}$ pour les deux modes de fonctionnement de la lentille. A nouveau, on constate que le mode accélérateur conduit à des valeurs de $G_{\mathrm{c}}$ nettement inférieures à celles obtenues pour la lentille fonctionnant avec la même distance focale en mode retardateur. Ainsi pour $f / D \simeq 2, G_{c}$ est neuf fois plus petit pour le premier mode que pour le second. Des résultats analogues sont obtenus pour d'autres géométries et d'autres points conjugués.

4. Conclusion. - Les résultats qu'on vient de donner montrent que les lentilles accélératrices devraient être de meilleure qualité. Des expériences récentes nous ont permis de vérifier qu'il en était bien ainsi. L'utilisation de ces lentilles est particulière-

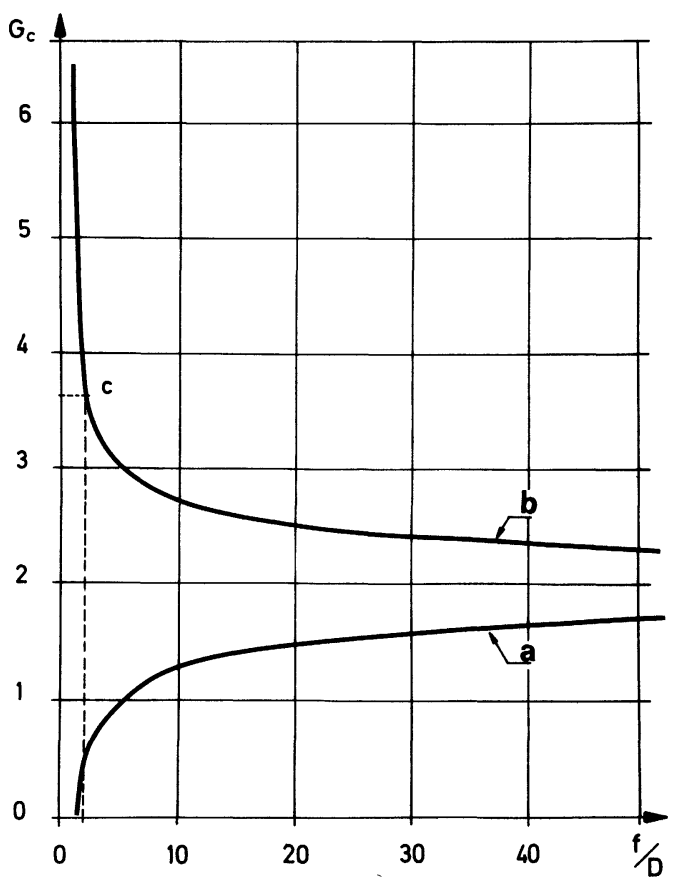

Fig. 4. - a : Mode accélérateur ; b : Mode décélérateur ; c : Limite de surexcitation $(v=0)$.

[a : Accelerating mode. b : Retarding mode. c : Overexcitation point $(v=0)$.]

ment recommandée lorsqu'il s'agit de focaliser des faisceaux polychromatiques comme c'est le cas, par exemple, pour des particules qui proviennent d'une émission secondaire. De même, la production de sondes fines pourrait bénéficier de la réduction de l'aberration d'ouverture. Pour ce dernier type d'application, il est à noter que nos résultats vont dans le même sens que ceux obtenus par Kuroda et Suzuki [6] bien que dans leur cas il s'agisse plutôt de systèmes post-accélérateurs.

L'énergie $V_{2}$ des ions qu'on cherche à focaliser peut cependant introduire une limite à l'utilisation de ce type de lentille. En effet, elle peut conduire à des potentiels d'accélération $V_{1}$ trop élevés, incompatibles avec la tenue en tension des lentilles. De plus, sur le plan pratique, on sera amené à utiliser deux sources de tension qui devront de ce fait être hautement stabilisées. Mais cet inconvénient sera compensé par une réduction d'effets parasites comme ceux liés à des champs de fuite magnétique (près d'un spectromètre de masse par exemple) ou encore comme ceux qui proviennent de charge sur les électrodes lorsqu'elles se recouvrent accidentellement de pellicules légèrement isolantes. 


\section{Bibliographie}

[1] HARTING, E. et ReAd, F. H., Electrostatic lenses, Elsevier (1976) p. 212.

[2] SEPTIER, Aberration sphérique de quelques lentilles électrostatiques à symétrie de révolution pour des faisceaux de grande ouverture CERN 60-39 octobre 1960.

[3] Regenstreiff, E., Thèse : Théorie de la lentille électrostatique à trois électrodes (ed. Gauthier-Villars, Paris) 1951.
[4] Zworykin, V. K. et coll., Electron optics and the electron microscope (John Wiley, New York) 1945, p. 604 et 572.

[5] El-Karreh, A. B. et Sturans, M. A., Analysis of a range of symmetrical three aperture electrostatic lenses, IEEE Trans. electron devices, October 1972.

[6] Kuroda, K. et Suzuki, T., J. Appl. Phys., Volume 45, no 3 (March 1974). 